\title{
THE UNIQUENESS OF THE NEAR HEXAGON ON 729 POINTS
}

\author{
A. E. BROUWER
}

Received 6 Augustus 1981

\begin{abstract}
We prove that any regular near hexagon with 729 vertices and lines of size 3 is derived from the ternary Golay code, thus settling the last case in doubt among the regular near hexagons with lines of size 3 .
\end{abstract}

\section{Introduction}

A near hexagon is a partial linear space $(X, \mathscr{L})$ such that

(a) For any point $p \in X$ and line $\ell \in \mathscr{L}$ there is a unique point on $\ell$ nearest $p$.

(b) Every point is on at least one line.

(c) The distance between any two points is at most three.

(The distances are measured in the point graph: $d(p, q)=1$ iff $p$ and $q$ are collinear.) A regular near hexagon with parameters $\left(s, t, t_{2}\right)$ is a near hexagon such that each line contains $1+s$ points, each point is in $1+t$ lines, and a point at distance 2 from a fixed point $x_{0}$ is in $1+t_{2}$ lines containing a neighbour of $x_{0}$.

Shult and Yanushka [4] showed that there are exactly eleven possibilities for the parameters of a regular near hexagon with $s=2$. For ten parameter sets the corresponding near hexagons have been classified completely (by Cameron, Shult and Yanushka, Tits, Cohen and Brouwer). Here we settle the last case by showing that there is a unique regular near hexagon with parameters $\left(s, t, t_{2}\right)=(2,11,1)$. As Shult and Yanushka indicate, an example is given by the 729 vectors of the extended ternary Golay code, where lines are triples $\{x, y, z\}$ with $x+y+z=O$ and $d_{H}(x, y)=$ $=d_{H}(x, z)=d_{H}(y, z)=12$ (where $d_{H}$ is the Hamming distance in $\mathbf{F}_{3}^{12}$ ). One finds that distance $0,1,2,3$ in the point graph corresponds to vectors at Hamming distance $0,12,6,9$, respectively.

Here we find another way to describe this near-hexagon in terms of the extended ternary Golay code, and prove that any regular near hexagon with $\left(s, t, t_{2}\right)=$ $=(2,11,1)$ can be obtained in this way. 


\section{Parameters}

Let $\mathscr{H}$ be a regular near hexagon with parameters $\left(s, t, t_{2}\right)=(2,11,1)$. Let $k_{i}=\left|\Gamma_{i}\left(x_{0}\right)\right|=\#\left\{x \mid d\left(x, x_{0}\right)=i\right\}$ for some fixed $x_{0} \in \mathscr{H}$. Then

$$
\begin{aligned}
& k_{0}=1 \\
& k_{1}=24 \quad(=s(t+1)) \\
& k_{2}=264 \quad\left(=k_{1} \cdot s \cdot t !\left(t_{2}+1\right)\right) \\
& k_{3}=440 \quad\left(=k_{2} \cdot s \cdot\left(t-t_{2}\right) /(t+1)\right)
\end{aligned}
$$

so that $v=\Sigma k_{i}=729$.

Diagram of the distance regular point graph:

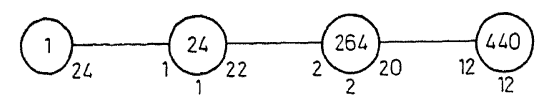

$\mathscr{H}$ is an association scheme with intersection numbers $\left(p_{i j}^{k}\right)$, where

$$
\begin{aligned}
&\left(p_{0 j}^{k}\right)_{j k}=\left(\begin{array}{llll}
1 & 0 & 0 & 0 \\
0 & 1 & 0 & 0 \\
0 & 0 & 1 & 0 \\
0 & 0 & 0 & 1
\end{array}\right),\left(p_{1 j}^{k}\right)=\left(\begin{array}{rrrr}
0 & 1 & 0 & 0 \\
24 & 1 & 2 & 0 \\
0 & 22 & 2 & 12 \\
0 & 0 & 20 & 12
\end{array}\right), \\
&\left(p_{2 j}^{k}\right)=\left(\begin{array}{rrrr}
0 & 0 & 1 & 0 \\
0 & 22 & 2 & 12 \\
264 & 22 & 131 & 78 \\
0 & 220 & 130 & 174
\end{array}\right), \quad\left(p_{3 j}^{k}\right)=\left(\begin{array}{rrrr}
0 & 0 & 0 & 1 \\
0 & 0 & 20 & 12 \\
0 & 220 & 130 & 174 \\
440 & 220 & 290 & 253
\end{array}\right) .
\end{aligned}
$$

Using some properties of $\mathscr{H}$ that will be proved in the next section we give here also the parameters w.r.t. a quad. If $Q_{0}$ is a fixed quad (cf. Shult and Yanushka [4]) then there are $9,180,540$ points at distance $0,1,2$ from $Q_{0}$, respectively. Diagram:

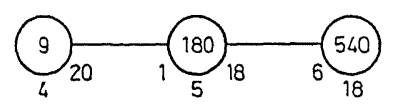

(A point in $Q_{0}$ is in 12 lines, 2 in $Q_{0}$ and 10 with one point in $Q_{0}$ and two points in $\Gamma_{1}\left(Q_{0}\right)$. A point in $\Gamma_{1}\left(Q_{0}\right)$ is in 12 lines, 1 meeting $Q_{0}, 2$ contained in $\Gamma_{1}\left(Q_{0}\right)$, 9 with two points in $\Gamma_{2}\left(Q_{0}\right)$. A point in $\Gamma_{2}\left(Q_{0}\right)$ is in 12 lines, 6 contained in $\Gamma_{2}\left(Q_{0}\right)$ and 6 having one point in $\Gamma_{1}\left(Q_{0}\right)$.) 


\section{Quads and cubes}

(For definition, existence and properties of quads, see [4].) In $\mathscr{H}$ the quads have 9 points and 6 lines and look like the picture shown, i.e., they are GQ $(2,1)$ 's. Any two intersecting lines determine a quad. We shall show that any three concurrent lines determine a $3 \times 3 \times 3$ cube (called $H(27)$ by Shult and Yanushka).

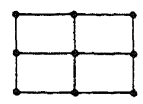

A point $x$ at distance one from a quad $Q$ has a unique neighbour in the quad, say $\pi_{Q}(x)$ or just $\pi(x)$. In any near hexagon the following is true:

Lemma. Let $Q$ be a quad and $x, y$ two adjacent points at distance one from $Q$, where $\pi(x) \neq \pi(y)$ (i.e., the line $x y$ does not intersect $Q$ ). Then

(i) any point on $x y$ has distance one to $Q$;

(ii) $\pi(x y)$ is a line in $Q$.

Proof. First of all $\pi(x) \sim \pi(y) \quad(\sim$ denotes adjacency, i.e., collinearity), since $d(x, \pi(y))=2$ and a shortest path from $x$ to $\pi(y)$ can be found through $\pi(x)$. If $z \in x y$ and $d(z, Q)=2$ then the collection $O$ of points in $Q$ closest to $z$ forms an oval in $O$ (i.e., a set of points in $Q$ meeting each line of $Q$ in exactly one point), but $\pi(x)$. $\pi(y) \in O$, a contradiction. This proves (i), and shows that $\pi(x y)$ is a clique in $Q$, so is contained in a line $\ell$. Let $p \in \ell$. Let $q$ be the point on $x y$ closest to $p$. Since there are two points on $x y$ at distance 2 from $p$ and $x y$ does not meet $Q$ it follows that $d(p, q)=$ $=1$, i.e. $p \in \pi(x y)$.

In our case there are 6 ovals in any quad - the quad together with (lines and ovals) as lines is an affine plane $\operatorname{AG}(2,3)$ - and the ovals fall into two parallel classes. Fix a quad $Q$ and call the two parallel classes // and $\|$. If $x$ and $y$ are two adjacent points in $\Gamma_{2}(Q)$ then either the line $x y$ is contained within $\Gamma_{2}(Q)$, and the ovals determined by $x$ and $y$ are disjoint, hence parallel, or the third point $z$ of $x y$ is in $\Gamma_{1}(Q)$, and the ovals determined by $x$ and $y$ meet in $\pi(z)$, hence are not parallel.

Fix a point $x \in \Gamma_{2}(Q)$ and consider the quads $Q^{\prime}$ containing $x$. These are of seven possible types indicated by a diagram: an open dot is a point of $Q$, a solid dot is in $\Gamma_{1}(Q)$ and $/ /$ and $\|$ label points belonging to ovals in these two parallel classes. We may assume that $x$ itself is labelled.
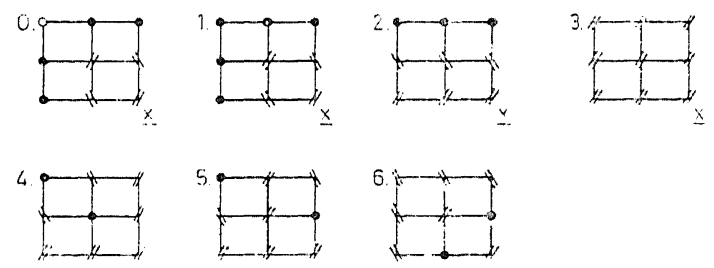

(Note that patterns with one or three solid dots (not on a line) are impossible. The former since it cannot be completed with $/ /$ and $\|$ labels. The latter because if $p, q, r$ are three points in $Q^{\prime} \cap \Gamma_{1}(Q)$ and $y$ is a common neighbour of $p$ and $q$ in $Q^{\prime}$ then the 
oval determined by $y$ in $Q$ contains $\pi(p)$ and $\pi(q)$ and therefore is $\{\pi(p), \pi(q), \pi(r)\}$; now $y$ must be adjacent ro $r$, a contradiction.)

In order to exclude possibility 1, let us write down some equations for $n_{i}$, the number of quads $Q^{\prime}$ of type $i$.

(a) Count lines $y z$ in $\Gamma_{1}(Q)$ with $x \sim y: X$ is in 12 lines, 6 contained in $\Gamma_{2}(Q)$ and 6 with a unique point in $\Gamma_{1}(Q)$. Thus we have 6 choices for $y$, and given $y$ we have 2 choices for the line $y z$. This shows that

$$
2 n_{1}+n_{2}=12 \text {. }
$$

(b) Count pairs of lines passing through $x$ and contained in $\Gamma_{2}(Q)$ :

$$
n_{3}+n_{4}=15 \text {. }
$$

(c) Count pairs of lines passing through $x$ and meeting $\Gamma_{1}(Q)$ :

$$
n_{0}+n_{1}+n_{6}=15 \text {. }
$$

(d) Count pairs at distance 2 from $x$ in $\Gamma_{1}(Q)$ : One one hand there are $2 n_{0}+$ $+3 n_{1}+2 n_{2}+2 n_{4}+n_{5}$ such points (for: such a point determines together with $x$ a unique quad $Q^{\prime}$ ); on the other hand, for points $z \in \Gamma_{1}(Q)$ with $d(x, z)=2$ consider $y=\pi(z)$. If $d(x, y)=2$ then there are $p_{21}^{2}=2$ points $z$ with $\pi(z)=y$. If $d(x, y)=3$ then there are $p_{21}^{3}-2=10$ points $z$ with $\pi(z)=y$. Altogether we find $3 \cdot 2+6 \cdot 10=$ $=66$ points $z$. Thus:

$$
2 n_{0}+3 n_{1}+2 n_{2}+2 n_{4}+n_{5}=66 .
$$

(e) Count points at distance 2 from $x$ in $Q$ :

$$
n_{0}=3 \text {. }
$$

(f) Count pairs of lines passing through $x$ :

$$
n_{0}+n_{1}+n_{2}+n_{3}+n_{4}+n_{5}+n_{6}=66 .
$$

This gives us six equations with seven unknowns. Putting $n_{1}=\alpha$ we find

$$
\begin{aligned}
n_{0}=3, n_{1}=\alpha, n_{2} & =12-2 \alpha, n_{3}=9+\frac{1}{2} \alpha, n_{4}=6-\frac{1}{2} \alpha \\
n_{5} & =24+2 \alpha, n_{6}=12-\alpha .
\end{aligned}
$$

Now put $\alpha=\alpha(x), n_{i}=n_{i}(x)$ and vary the point $x$. Clearly, each quad $Q^{\prime}$ with 2 points in $\Gamma_{1}(Q)$ is of type 4 for one point $x$, of type 5 for four points $x$ and of type 6 for two points $x$. Consequently, averaging over $x$, we have $\bar{n}_{5}=4 \bar{n}_{4}$ and $\bar{n}_{6}=2 \bar{n}_{4}$, so that $\bar{\alpha}=0$. But if $n_{1}$ is zero on the average then it is always zero, and we have for each $x$ :

$$
n=(3,0,12,9,6,24,12) .
$$

In particular case (1) does not occur, so that we proved: 
Lemma. Let $Q, Q^{\prime}$ be two points such that $Q^{\prime} \cap \Gamma_{1}(Q)$ contains two intersecting lines. Then $Q^{\prime} \subset \Gamma_{1}(Q)$.

Now we are in a position to prove the announced existence of cubes:

Proposition. Let $\ell_{1}, \ell_{2}, \ell_{3}$ be three concurrent lines. Then there is a unique minimal set $C$ containing these three lines and such that if it contains two points at distance 2 then also the quad determined by them. We have $|C|=27$ and the graph induced on $C$ is isomorphic to the graph on the Hamming scheme $\mathbf{F}_{3}^{3}$ where adjacency $\equiv$ Hamming distance one. Such sets $C$ are called cubes.

Proof. Let $Q_{i j}=\left\langle\ell_{i}, \ell_{j}\right\rangle$ be the quad defined by $\ell_{i}$ and $\ell_{j} \quad(i, j=1,2,3 ; i \neq j)$. Let $C=Q_{12} \cup Q_{13} \cup\left\{y \mid d\left(y, Q_{12}\right)=d\left(y, Q_{13}\right)=1, d\left(y, \ell_{1}\right)=2\right\}$. Then $Q_{23} \subset C$, and if $y \in C \backslash\left(Q_{12} \cup Q_{13} \cup Q_{23}\right)$ and $z \in \ell_{1}$ with $d(y, z)=2$ then by the lemma above each point of the quad $Q(y, z)$ has distance one to $Q_{23}$. Using the fact that if $Q$ is a quad and $x \in \Gamma_{1}(Q)$ there are exactly two lines passing through the point $x$ and contained entirely within $\Gamma_{1}(Q)$ one easily verifies all claims.

\section{Vector space structure}

Assuming the validity of this proposition Shult and Yanushka proved the existence of a regular abelian group of automorphisms of $\mathscr{H}$. In fact they first proved (tediously):

Let $d(x, y)=3$. Then there are precisely 4 cubes containing both $x$ and $y$. These cubes meet, except for $x$ and $y$, in a unique third point $z$, where $z$ is the unique point at distance 3 from both $x$ and $y$ in each cube.

Now fixing a vertex and calling it $\underline{0}$ we may define an involution $x \rightarrow-x$ by fixing $\underline{0}$, interchanging points $\neq 0$ on a line through $\underline{0}$, interchanging points $\neq 0$ on an oval through $\underline{0}$, and interchanging points $y, z$ if $\underline{d}(\underline{0}, y)=d(\underline{0}, z)=d(y, z)=\overline{3}$, and $z$ is in each of the 4 cubes containing $\underline{0}$ and $y$.

The product of two such involutions (with different fixpoints) is an automorphism without fixpoints and is uniquely determined by the image of some vertex $\underline{0}$. These automorphisms are our translations, and we have the group $\mathbf{Z}_{3}^{6}$.

Identify the vertices with group elements. Now we have given $\mathscr{H}$ the structure of a vector space over $\mathbf{F}_{3}$, and lines and ovals have the property that the sum of their elements is zero. Lines, quads and cubes are one-, two- and three-dimensional subspaces (but there are also other subspaces of these dimensions). [Our subspaces are affine subspaces - they need not contain $\underline{0 .}$.]

\section{Buekenhout diagram}

At this point I did not know how to continue: the extended ternary Golay code is a 6-dimensional subspace of a 12-dimensional vector space over $\mathbf{F}_{3}$. We found the subspace, but where is the big space? One would like to identify the 12 coordinate positions, and an obvious choice seems to be the set of twelve lines through $\underline{0}$. But in Shult and Yanushka's construction these lines do not correspond to coordinate positions. A coordinate position gives us a coclique of size 243, but it is not clear how 
to find such cocliques. Arnold Neumaier suggested first to identify the objects belonging to the Buekenhout diagram

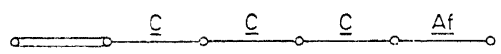

of the extended Golay code, and indeed, these are easy to find. But having found these one may define a Golay code where the 12 coordinate positions are in fact the 12 lines through $\underline{0}$. Thus we find a new construction of the near hexagon $\mathscr{H}$ and prove that any near hexagon with these parameters can be obtained in this way.

So, let us look at the diagram. The first three species are points, lines and quads; the next type is formed by the cubes. Objects $F$ of the fifth type have the property that any two cubes with a quad in common determine a unique such $F$. So these must be hypercubes, generated by four concurrent lines. (We shall call our objects $i$-spaces $(i=0,1,2,3,4,5)$; note that not each subspace of dimension $i$ is an $i$-space.)

We must show that the 4-space $F$ generated by four lines (of $\mathscr{H}$ ) through a point $x_{0}$ cannot contain other lines (of $\mathscr{H}$ ). Two cubes with a quad in common cannot meet outside this quad since the intersection of subspaces is a subspace. Thus we find that the four cubes determined by the triples from the four given lines contain together 65 points $\left(1,8,24,32\right.$ points at distance $0,1,2,3$ from $x_{0}$, respectively) so that $F$ contains 16 more points. If we set $x_{0}=\underline{0}$ then these points are sum of four vectors along the four given lines, but each sum of these three vectors has distance 3 to $\underline{0}$ so our new points have distance at least 2 to $\underline{0}$ (in fact 8 are at distance 2 and 8 at distance 3 ), and all points at distance 1 from $\underline{0}$ in $F$ lie on the four given lines.

The last type of object is determined by two 4-spaces with a 3-space in common, hence must be a 5 -space generated by five lines through a point $x_{0}$. The stroke denotes an $\mathrm{AG}(2,3)$ in this case, so we want to prove (i) that this partial linear space has 9 points - and indeed, given a cube containing $x_{0}$ there are 94 -spaces $F$ containing this cube, determined by the 9 lines on $x_{0}$ not in the cube, and (ii) that this partial linear space has lines of size 3, i.e., that 5-spaces generated by five lines through $x_{0}$ contain in fact six lines through $x_{0}$.

Suppose that the 5 -space $G$ on $\underline{0}$ contains only 5 lines (of $\mathscr{H}$ ) through $\underline{0}$, i.e. only 10 points at distance one from $\underline{0}$. The point set of $\mathscr{H}$ is partitioned by $\bar{G}$ and its two cosets $G_{1}$ and $G_{2}$. Since $k_{1}=\overline{2} 4$ there is a coset, say $G_{1}$, containing at least 7 oints at distance one from $\underline{0}$. $G_{1}$ is union of three 4-spaces, at least one of which intains 3 points at distance one from $\underline{0}$. Now we find a contradiction from

mma. Let $F$ be a 4-space. Then a point $x \notin F$ has at most 2 neighbours in $F$.

sof. Suppose $x$ has three neighbours in $F$. If $C$ is a cube contained in $F$ then $x$ has cisely one neighbour in $C$ (not more since a cube is a Cameron set, i.e., is closed der formation of quads - not less since $F$ is union of $C$ and two translates of $C$ ). Jordinatize $F$ with $\mathbf{Z}_{3}^{4}$. Then the 3 points in $F$ at distance 1 from $x$ have no coordiate in common so are 1111, 2222 and 0000 , say. Then $x$ is at distance 2 from 1000, 011 in the quad $10^{* *}$, hence also from 1022 . Looking at the line ${ }^{*} 022$ we have two points, 1022,2022 at distance 2 from $x$, so that 0022 must be at distance 1 from $x$, a contradiction. 
Consequently, $G$ contains at least 6 lines through 0 , and the linear space of 4-spaces and 5-spaces on a fixed 3-space has lines of size at least 3 . But there are only two linear spaces on 9 points with lines of size at least 3 , namely AG $(2,3)$ and the space with a single line of size 9 . The latter, however, is impossible - if $G$ contains all lines through $\underline{0}$ then $G$ contains all of $\mathscr{H}$ and cannot be a 5-space.

This proves that we indeed have the diagram given above.

\section{Uniqueness}

It is well-known that there is a unique object with diagram $\underline{C}_{0} \underline{C}_{0} \underline{C}_{0} \underline{\Delta F_{0}}$ where Af denotes an $\operatorname{AG}(2,3)$, namely the Witt design $\mathrm{S}(5,6,12)$. We use only the simple property that the complement of a block of this design again is a block. Choose six lines $\ell_{i}(0 \leqq i \leqq 5)$ through $\underline{0}$ not forming a block in the local design $\mathrm{S}(5,6,12)$. Then the remaining six lines $\ell_{i}(6 \leqq i \leqq 11)$ do not form a block either. Choose a point $e_{i} \neq 0$ on each of the lines $\ell_{i}$. Then both $B=\left\{e_{i} \mid 0 \leqq i \leqq 5\right\}$ and $B^{\prime}=\left\{e_{i} \mid 6 \leqq i \leqq 11\right\}$ form a basis for the vector space $\mathscr{H}$. Associate a vector of length 12 with each point $x \in \mathscr{H}$, writing $x \mapsto\left(\alpha_{0}, \ldots, \alpha_{11}\right)$ if $x=\sum_{i=1}^{5} \alpha_{i} e_{i}=\sum_{i=6}^{11} \alpha_{i} e_{i}$, The image of $\mathscr{H}$ under this map is a 6-dimensional subspace of $Z_{3}^{12}$. Let us prove that it is the extended Golay code. To this end it suffices to show that the minimum distance is 6 (cf. Delsarte and Goethals [2], Pless [3]).

Suppose $v=\left(\alpha_{0}, \ldots, \alpha_{11}\right)$ is a nonzero vector of weight at most 5. Let $w_{L}(v)=$ $=\#\left\{i \mid \alpha_{i} \neq 0\right.$ and $\left.0 \leqq i \leqq 5\right\}$ and $w_{R}(v)=\#\left\{i \mid \alpha_{i} \neq 0\right.$ and $\left.6 \leqq i \leqq 11\right\}$ be the left and right weights of $v$. Then $v$ can be written as sum of $w_{L}$ multiples of vectors in $B$ and also as sum of $w_{R}$ multiples of vectors in $B^{\prime}$. Therefore there is a representation of $\underline{0}$ as linear combination with nonzero cooefficients of $w=w_{L}+w_{R}$ vectors in $B \cup B^{\prime}$, or also a representation of one vector in $B \cup B^{\prime}$ as linear combination of at most four others. But we know that the 4-space generated by these others does not contain other points of $B \cup B^{\prime}$, a contradiction.

This proves that the image of $\mathscr{H}$ is the extended Golay code. But conversely, if $\mathscr{C}$ is the extended Golay code then let $D \subset \mathscr{C}$ be the set of 24 vectors with exactly one nonzero coordinate in the first 6 positions or exactly one nonzero coordinate in the last 6 positions. We find $\mathscr{H}$ back again by calling two vectors adjacent if their difference belongs to $D$. (Now the lines of $\mathscr{H}$ are the triangles in the point graph.)

Note that we obtain only one near hexagon $\mathscr{H}$ in this way (up to isomorphism): the automorphism group of $\mathscr{C}$ contains $M_{12}$ acting on the positions (cf. [1], page 85), and under this group the 6-sets fall into two orbits: those corresponding to a block of $S(5,6,12)$, i.e., those forming the support of a codeword, and the remaining ones. Clearly, when talking about 'the first six positions' we mean a 6-set not forming a block. (Note: here automorphisms are monomial transformations, i.e., matrices with one nonzero entry in each row and column. That there are only two orbits, is seen as follows: $M_{12}$ is sharply 5-transitive and the setwise stabilizer of a 5-set has order 120; it fixes a 6 th point and acts as PGL $(2,5)$ on the remaining 6 points.) Thus there is a unique regular near hexagon with parameters $\left(s, t, t_{2}\right)=(2,11,1)$. 


\section{References}

[1] P. J. Cameron and J. H. VAN Lint, Graph Theory, Coding Theory and Block Designs, LMS Lecture Notes 19, Cambridge Univ. Press, Cambridge, 1975.

[2] Ph. Delsarte and J. M. Goethals, Unrestricted codes with the Golay parameters are unique, Discr. Math. 12 (1975) $211-224$.

[3] V. PLESS, On the uniqueness of the Golay codes, J. Comb. Theory 5 (1968) 215-228.

[4] E. E. Shult and A. YanushKa, Near $n$-gons and line systems, Geometriae Dedicata 9 (1980) $1-72$.

A. E. Brouwer

Mathematisch Centrum

Kruislaan 413, 1098 SJ Amsterdam

The Netherlands 